This item was submitted to Loughborough's Research Repository by the author.

Items in Figshare are protected by copyright, with all rights reserved, unless otherwise indicated.

\title{
Observations on the implementation of LEDs for general lighting
}

PLEASE CITE THE PUBLISHED VERSION

http://dx.doi.org/10.1109/ITHERM.2016.7517634

PUBLISHER

(c) IEEE

VERSION

AM (Accepted Manuscript)

\section{PUBLISHER STATEMENT}

This work is made available according to the conditions of the Creative Commons Attribution-NonCommercialNoDerivatives 4.0 International (CC BY-NC-ND 4.0) licence. Full details of this licence are available at: https://creativecommons.org/licenses/by-nc-nd/4.0/

\section{LICENCE}

CC BY-NC-ND 4.0

\section{REPOSITORY RECORD}

Pryde, James R., David C. Whalley, and W. Malalasekera. 2019. "Observations on the Implementation of Leds for General Lighting". figshare. https://hdl.handle.net/2134/21806. 


\title{
Observations on the implementation of LEDs for general lighting
}

\author{
James R. Pryde \\ Tamlite Lighting \\ Tamlite Field Sales Centre, Park Farm Industrial Estate, Redditch, Worcestershire, B98 0HU, United Kingdom \\ $\&$ \\ Loughborough University, Wolfson School of Mechanical and Manufacturing Engineering \\ Loughborough, Leicestershire, United Kingdom, LE11 3TU, United Kingdom \\ Telephone: +447757 69070 \\ Email: j.r.pryde@lboro.ac.uk \\ David. C. Whalley, Weeratunge Malalasekera \\ Loughborough University, Wolfson School of Mechanical and Manufacturing Engineering \\ Loughborough, Leicestershire, United Kingdom, LE11 3TU, United Kingdom
}

\begin{abstract}
LEDs offer a number of advantages over existing light source technologies. For this reason they have been extensively developed and adopted by the general lighting industry. However, the array of LED types and luminaire designs is vast and the existing academic literature provides no known assessment as to which are most relevant to current or future industrial practices. Consequently it is unclear how the associated thermal management strategies are expected to develop. This paper set out to address this gap. Market surveys were conducted in 2013 and 2015 to obtain data on commercially available luminaires. This survey captured details of the LED type and thermal management strategy employed. The lead author's position as a design engineer with a UK based luminaire manufacturer was also used to provide insight into market factors and demands which influence industry practice. The findings of this work suggest market influences will favour mid-power LEDs in future products for the simpler luminaire design and greater lifecycle performance they offer. In general, dedicated thermal management appeared to be becoming less critical to luminaire design although there were still numerous examples of its relevance.
\end{abstract}

Key words: market survey, trends, luminaire design, system integration, thermal management

\section{Introduction}

Light Emitting Diodes (LEDs) offer various advantages over existing light source technologies. Their superior luminous efficacy, greater service life and versatility are widely known. Ongoing increases in luminous flux per die [1] have enabled them to become a viable and cost effective alternative to established technologies. LED's are projected to account for $63 \%$ of a potential global lighting market worth $\$ 100 \mathrm{bn}^{(\mathrm{US})}$ by 2020 [2] making them an extremely valuable technology. However, the performance and reliability of the LED remains critically linked to its thermal environment during operation [3]. Consequently thermal management of the LED is an integral part of luminaire design.

Due to their advantages, the proliferation of LED's in the general lighting industry has been rapid. The result is a wide array of component package styles and performance categories with unique benefits and limitations. The objective of this paper was to address some apparent gaps in the existing academic literature i.e. to identify which of these categories are most relevant to industry practices, how industry practice appears to be evolving, and consequently how can this evolution be expected to influence the luminaire's thermal management design. This study provides the foundation to a wider doctoral thesis exploring the effective thermal management of LEDs and similar technologies.

To address the objectives of this study an overview of the industry was required. The lead author's work as a design engineer within a UK based luminaire manufacturer provided an insight into commercial product development practices. Observations from this perspective were offered on the current status of the industry. These observations were combined with market survey data. The survey captured published data from a selection of luminaire manufacturers regarding which types of LED they employ in their products and how their thermal management was catered for. The findings were analysed to identify industry trends in luminaire design and the implementation of LEDs.

\section{Survey methodology}

A range of manufacturer's products were surveyed in an attempt to capture a broad overview of industry practice. Their selection was performed without any commercial influence or conscious bias or towards particular organisations aside from the availability of data. Information was only recorded when explicitly provided in the manufacturer's published literature (i.e. datasheets, product leaflets, catalogues, etc.). Under no circumstances were assumptions made. The only exception to these conditions was regarding data on Thorlux's products. This was obtained while the lead author was in their employment. It was supplied directly by the company's technical manager and verified as accurate by the author.

The parameters of interest were the luminaire's total luminous flux, LED component package type, LED component package power consumption, luminaire thermal management strategy, forming processes employed and material composition. The luminaires are grouped according to the LED type 
employed. The LEDs were categorised as either low power ( $<$ $0.1 \mathrm{~W})$, medium power $(0.1<1 \mathrm{~W})$, high power $(>1 \mathrm{~W})$ or a chip-on-board (COB) array. In the context of this survey, and following industry convention, $\mathrm{COB}$ array refers solely to the package style shown in Fig. 1 rather than defined by a particular power consumption range. The nature of these modules permits a wide variety of operating powers with examples as high as 175 $\mathrm{W}$ [4] readily available.

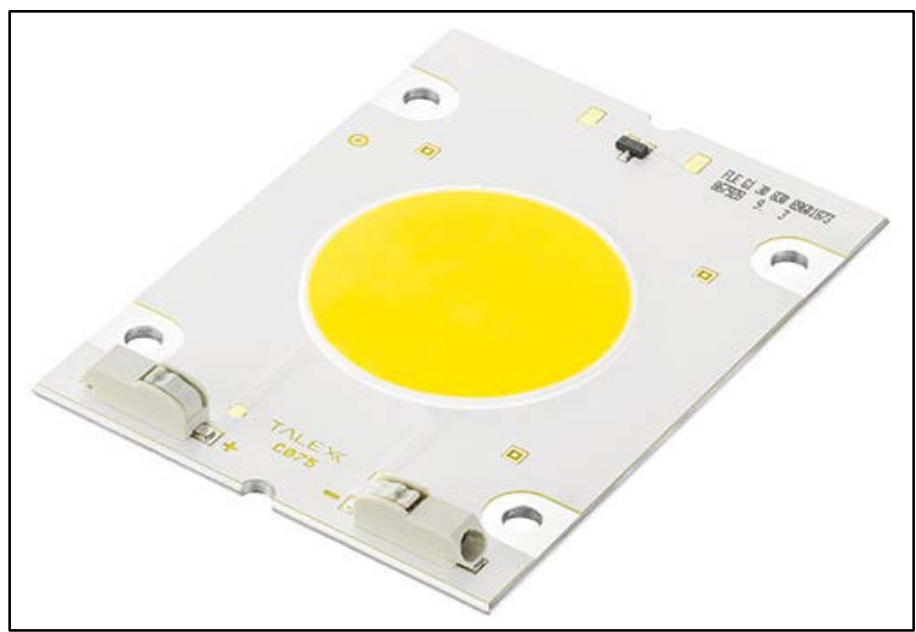

Fig. 1: An example of a COB array [4]

In order to evaluate how the implementation of LEDs is evolving as the industry matures surveys were conducted at two distinct points in time, the first during October, 2013 and the second during October, 2015. The original survey formed part of an earlier review conducted by Pryde et al. [5]. Future surveys to expand the timeframe of this evaluation would be extremely useful but unfortunately not practical within the confines of this study. The first survey drew from each company's entire catalogue of available products. The second survey only included new products launched in the interim period. The first survey sample size was 75 , while the second was 76 . In cases where multiple operating specifications of the same luminaire existed, only the highest power consumption, smallest volume model was recorded. For consistency the chosen sample emitted light at a colour temperature of $4000 \mathrm{~K}$ and with a colour rendering index $(\mathrm{CRI})>80$. Where this was not offered the closest alternative was recorded. It was assumed this compromise had a negligible effect on the results.

The lead author's experience as a luminaire design engineer and involvement with the industry provides a foundation to offer supporting observations. The influence of industry demands and market factors guiding LED luminaire development will be discussed alongside the survey findings.

\section{3 survey results}

The total luminous flux of each sample luminaire is plotted in Fig. 2. With respect to the range of luminous flux output by each luminaire $(100-20000 \mathrm{~lm})$, around $80 \%$ of products were clustered across a relatively narrow range $(300-3000 \mathrm{~lm}$ output). This luminous flux output appeared to cater for the majority of applications. There were a number of niche products offering higher and lower output but they represent a far smaller proportion of the market. Luminaires developed around high power LED packages dominated the data but there were some mid-power and COB array based products which offered comparable output. There were no cases of luminaires employing low power LEDs captured in the survey.

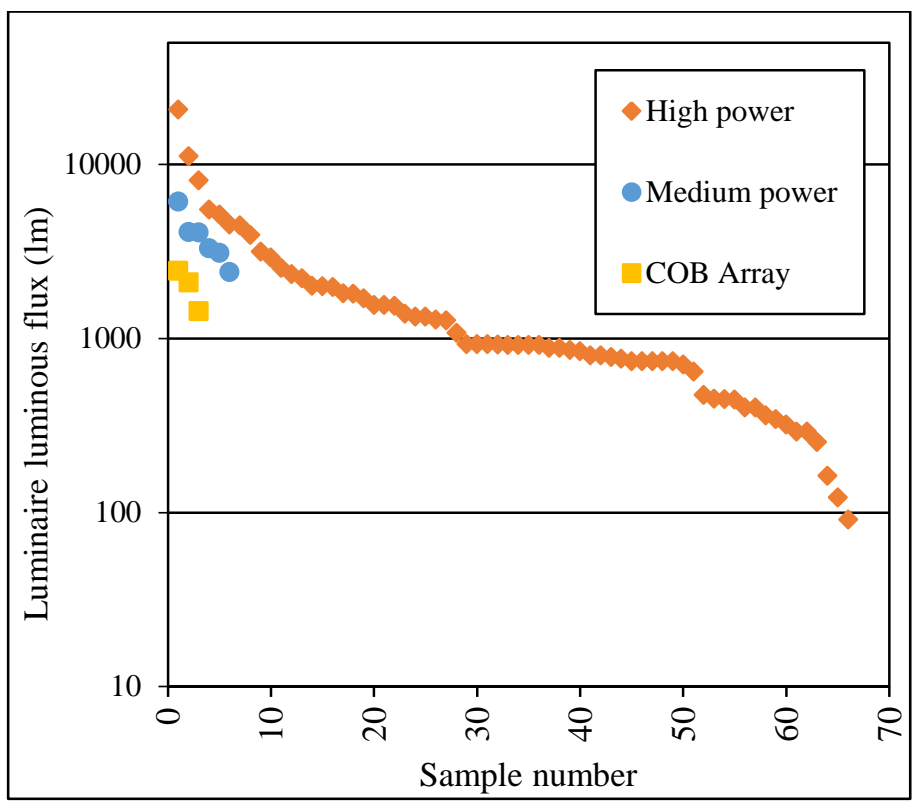

Fig. 2: 2013 survey, luminaire luminous flux differentiated by LED category.

The thermal management method employed by each of the sampled luminaires is summarised in Fig. 3. There were three methods observed; passive (natural convection) heatsinks (a dedicated structure with a large surface area to enhance heat transfer to the environment); body redistribution (no evidence of dedicated thermal management beside the structure of the luminaire's inherent heat transfer properties); and active (forced convection) cooling (augmented with additional systems such as electromechanical fans). These were divided according to the LED type they were employed alongside. Thermal management of high power LED luminaires showed they predominantly employ a passive heatsink. A small percentage adopted active cooling or had no dedicated thermal management components. Heatsinks were typically formed from extruded or die cast aluminium. Half of the mid-power LED luminaires surveyed employed no dedicated thermal management. Because these chips operate at lower power it is the author's belief that the waste heat released by each component was small enough to circumvent any requirement for dedicated management. However, this survey did not verify such a strategy provides adequate thermal management. An equal proportion of COB array based luminaires employ no dedicated thermal management. These LED modules are generally compact and dissipate more power, concentrating waste heat to the extent that dedicated thermal management was expected. Again, this study 
did not evaluate the suitability of the luminaire design. It should also be noted that the COB array and mid-power LED categories include only a small number of samples, which may have allowed the results to be skewed by atypical designs or errors.

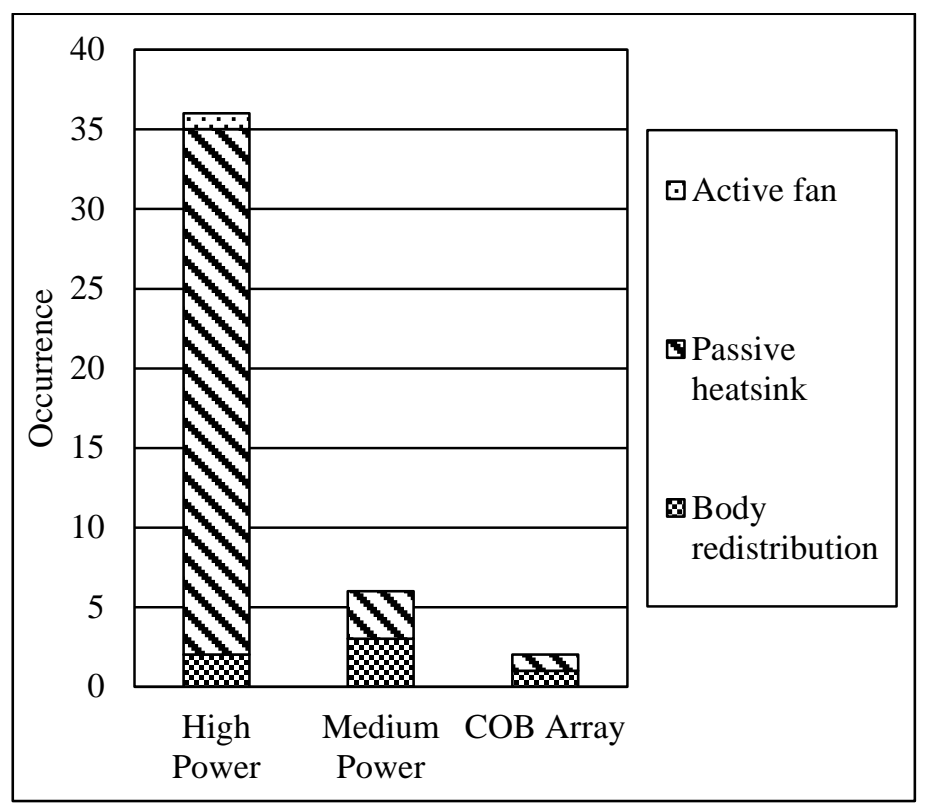

Fig. 3: 2013 survey, distribution of thermal management methods employed by each LED type.

\section{5 survey results}

This data was analysed in the same manner as for the previous survey. The luminous flux of each luminaire, differentiated by LED type employed, is plotted in Fig. 4, while the occurrence of each thermal management technique employed by the luminaires is plotted in Fig. 5 .

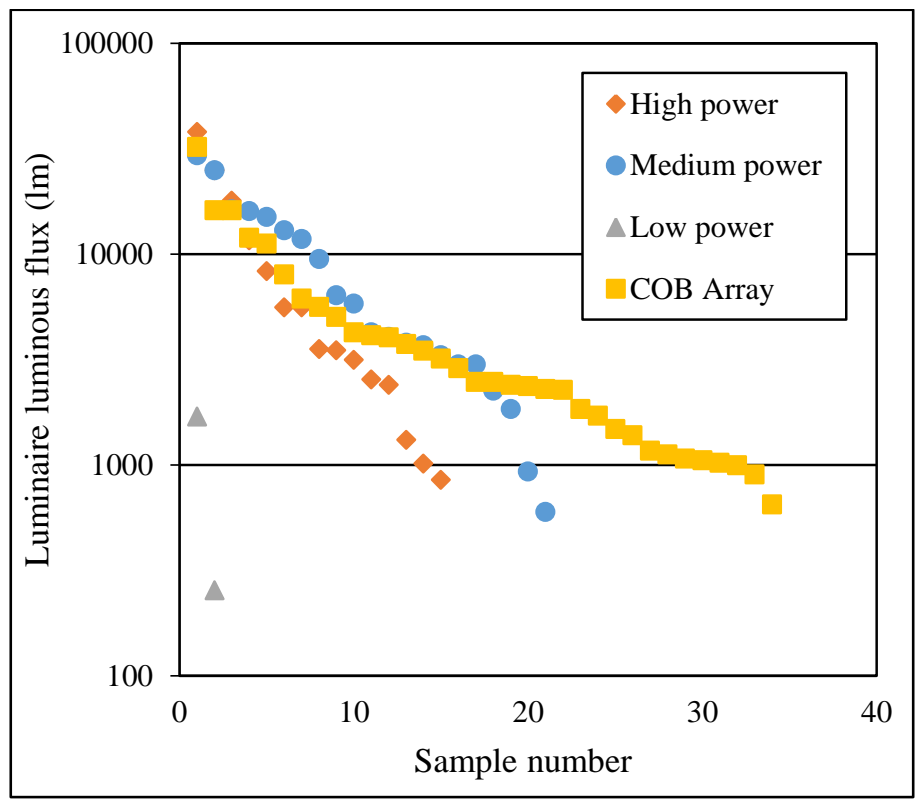

Fig. 4: 2015 survey, luminaire luminous flux differentiated by LED category.

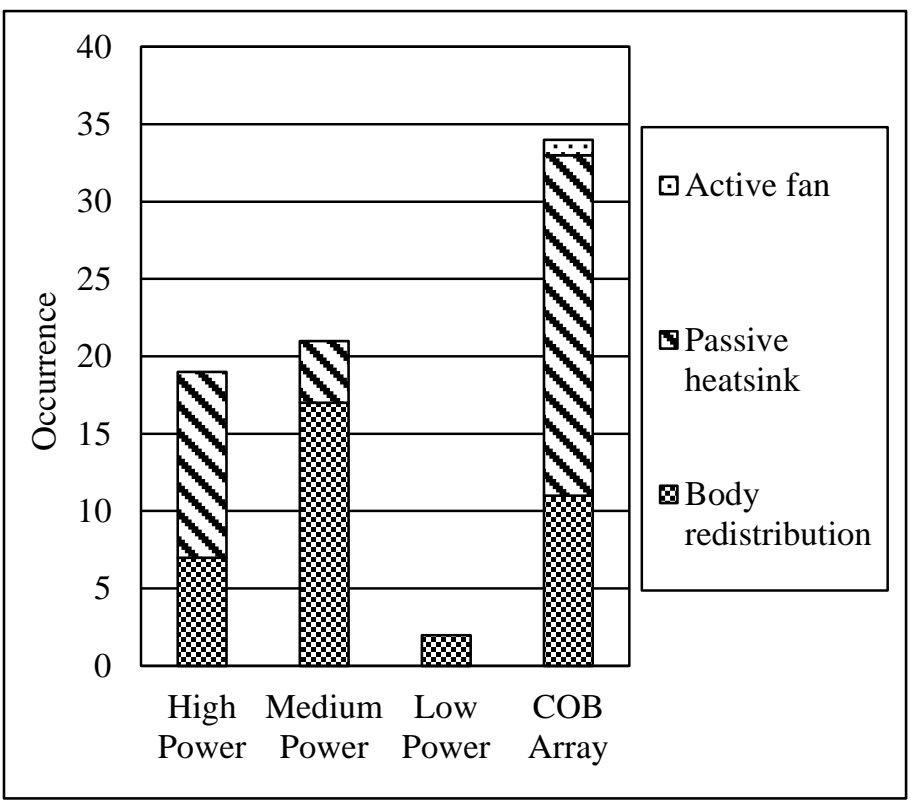

Fig. 5: 2015 survey, distribution of thermal management methods employed by each LED category.

The range of luminous flux from the surveyed luminaires $(250-38000 \mathrm{~lm})$ was higher than in the previous survey. The predominant luminous flux reflects literature comments that an ideal luminous flux 'sweet spot' of around $3000 \mathrm{~lm}$ [6] exists. Compared to the previous survey there were fewer samples in the low luminous flux $(<300 \mathrm{~lm})$ range.

In comparison to the 2013 survey, by 2015 there was far more data available for low, mid and COB type LEDs. COB LED arrays were the largest category of light source represented in this survey. These components tend to be more compact, offering improved aesthetics and permitting smaller luminaire designs. Mid-power LED based luminaires were also far more numerous in this survey, suggesting that they offer some commercial benefit which has driven their increase. There were two low power LED based luminaires identified, which represents an increase from the previous survey, but of limited significance. The greater quantity of low power LEDs required to deliver equivalent luminous flux to the alternatives may provide beneficial photometric characteristics or lower cost. However, the small number of luminaires employing these components suggest they offer limited commercial advantages over the alternatives and so were restricted to niche applications. Therefore this LED category appears to present a negligible potential influence on the general lighting industry.

In comparison to the previous survey a larger proportion of the high power LED and COB array based luminaires avoid dedicated thermal management. The proportions of each thermal management method were consistent for both LED categories, as was expected, due to the similar thermal conditions both package types establish. It was clear that many high power and COB array based luminaires still require some form of heatsink to establish suitable operating conditions. Examples taken from the raw data indicate the thermal management strategy 
expressed less association with luminaire output than in the previous survey. One actively cooled luminaire emitted just $2880 \mathrm{~lm}$ while many of the highest output luminaires incorporated no dedicated thermal management. These observations indicate the thermal management method was primarily dictated by the LED component category and form of the luminaire rather than the magnitude of heat transfer.

The heatsink materials and manufacturing methods employed were similar to those seen in the previous study. The majority of mid-power and all low power LED based luminaires employed no dedicated thermal management. With respect to mid-power LED based luminaires, the sample size was large enough to ensure an accurate representation of industry practice. Again, the findings do not prove specific thermal management measures were unnecessary. However, this appeared to be an increasingly common configuration and so must presumably be commercially viable.

\section{Limitations}

There were several issues with this analysis which need to be highlighted before evaluating the findings:

- $\quad$ The categorisation of LED types was based on an extremely simple and crude definition which left some ambiguity. Some of the reported high power LEDs may have employed multiple chips within a single package, essentially making them COB arrays, whereas industry tends to use the term COB array in reference to a particular style of module. Consequently the results may under report the proportion of luminaires actually using COB arrays. The impact on the findings is not believed to be significant. If anything this lessened the observed trend of growth in the use of COB arrays, however it is clear that COB has still become much more common in recent products.

- It was assumed that the surveys provide a fair representation of the industry, but many products, across multiple manufacturers' ranges, had to be excluded due to missing or unavailable data. This was an industry wide issue so was not believed to have unfairly influenced the findings. Its effect was believed to be equal for both surveys, so was not believed to have had any influence on the findings.

- The initial survey drew from each manufacturer's entire product catalogue, while the latter survey was limited to products released in the interim period. This means the samples of the initial survey do not necessarily correspond to that particular time period, distorting the observed pace of any development and preventing historical trends from being incorporated into the work. The sources of information rarely provided clear product history to avoid these issues. Consequently it was not possible to evaluate the rate at which the industry evolves.

- The data presented by suppliers was predominantly contained within their marketing material. This would tend to emphasise certain features over others. For instance, COB arrays in particular are a distinctive light source so are commonly highlighted, whereas low and mid-power LEDs are rarely identified. This would have influenced the relative proportions of sample products found under each category. To the authors' knowledge there was no reason to believe the emphasis of published data changed between surveys. Therefore the general growth/contraction of each category can be established, but any comparison between the relative size of each category would be invalid.

- The manufacturer's published data tended to lack document references, independent corroboration or consistency. This introduces considerable uncertainty, makes results difficult to reproduce, and hinders verification. Every effort was made to manage the quality of the data and any errors have to be assumed to be reasonably consistent (although the effect on smaller sample groups may have created some anomalous results). With these issues in mind, the findings of this work can only be treated as a very general assessment.

\section{Discussion}

Comparison of the results of the two surveys showed high power LED based luminaires being superseded by alternative LED types. The increased occurrence of mid-power and COB array based luminaires in the later survey suggest they offer commercial advantages which make them preferable to high power LEDs. At the present time high-power LEDs lag behind mid-power components in terms of luminous efficacy and also behind the compact, high luminous flux of COB arrays. Samsung's current high power LED, for instance, can emit 457 $\mathrm{lm}$ at a luminous efficacy of $98 \mathrm{~lm} . \mathrm{W}^{-1}$ [7]. Samsung's midpower component on the other hand can produce $69 \mathrm{~lm}$ at a luminous efficacy of $149 \mathrm{~lm} . \mathrm{W}^{-1}$ [8] while Tridonic's COB array [4] can produce $16,800 \mathrm{~lm}$ at a luminous efficacy of $96 \mathrm{~lm} . \mathrm{W}^{-1}$. It is believed these advantages have driven the increase in midpower and COB LED based luminaires and that the impact on luminaire design trends can be expected to continue. It should be noted that it is extremely difficult to report current performance benchmarks of each LED category in a meaningful and comparable manner. Not only do the properties of each vary widely depending on the operating conditions and between manufacturers, but rapid technological developments also mean they are continually improving. Therefore the figures quoted here are only provided as a general indication. Regarding lifetime, data for the different LED categories is difficult to obtain. Due to their long operating life the industry commonly uses the methods defined by TM-21-11 [9] to make predictions from short term test data. However, this still requires a 6000 hour test period. The rate of LED development means this information is nearly obsolete by the time it is available. It is believed this limits its usefulness and hence it is only provided for a small selection of components. The few sources available indicate lifetimes of the high power, mid-power and COB modules are all in excess of 50,000 hours [4][10][11].

Awareness of luminaire lifecycle performance and environmental impact appears to be a growing market influence, illustrated by a major manufacturer taking steps to provide environmental performance declarations (EPDs) for all of its 
products [13]. According to various lifecycle analyses (e.g. [14]), increasing luminous efficacy has the greatest potential to reduce the luminaire's environmental impact. Reducing material consumption also plays a significant role. Mid-power LEDs appeared to impose less demanding thermal management requirements (i.e. less heatsink material content) alongside superior luminous efficacy. It is the author's opinion that as LED technology matures and the market becomes saturated, differentiating superior products from competitors will become increasingly challenging. Highlighting environmental and lifecycle performance offers the means to address that challenge, which further supports the adoption of mid-power LEDs.

The review of academic literature by Chang et al. [3] shows agreement that reducing the LED's operating temperature is beneficial to its performance. However, the present survey results seemed to suggest integrating dedicated features to enhance heat transfer from the luminaire is becoming less common and almost totally absent when employing low or midpower components. The associated costs and complexity presumably outweigh any performance benefits. It also hints that LEDs are becoming more tolerant to high temperatures, thereby circumventing the need for dedicated thermal management. To achieve the greatest lifecycle performance this would appear to be an incorrect strategy. However, the increasing abundance of products omitting dedicated thermal management indicate that this approach is commercially feasible and advantageous. The potential cost reductions, simplified system design and reduction in material content that this permits are clearly valuable factors and would support the future growth of this strategy.

The later survey captured very few examples of luminaires which emit low luminous flux. It is the authors' belief there was limited justification to develop new products which satisfy a relatively small segment of the market, and for which a number of existing luminaires (as identified in the earlier survey) were already available. It appears the focus of commercial product development has shifted to higher output systems. By superimposing the two sets of survey data with the LED categories combined together (see Fig. 6) it is clear there has been an overall general upward shift in luminaire output occurring alongside developments in thermal management strategy. The observed trend for increased output seems to have no influence on thermal management strategy, instead luminaire design seems to be evolving to accommodate preferred thermal management strategies and LED types independently of luminaire output.

Low power LEDs appear to have very little bearing on the general lighting industry. There was limited evidence to suggest the growth of this category should be expected and no obvious advantages to drive any change.

As it currently stands there is limited demand for enhanced heat transfer from low and mid-power LED based luminaires. As these systems seem to be displacing high power LED based luminaires, any incentive to develop superior thermal management strategies for high power LED systems is also expected to diminish.

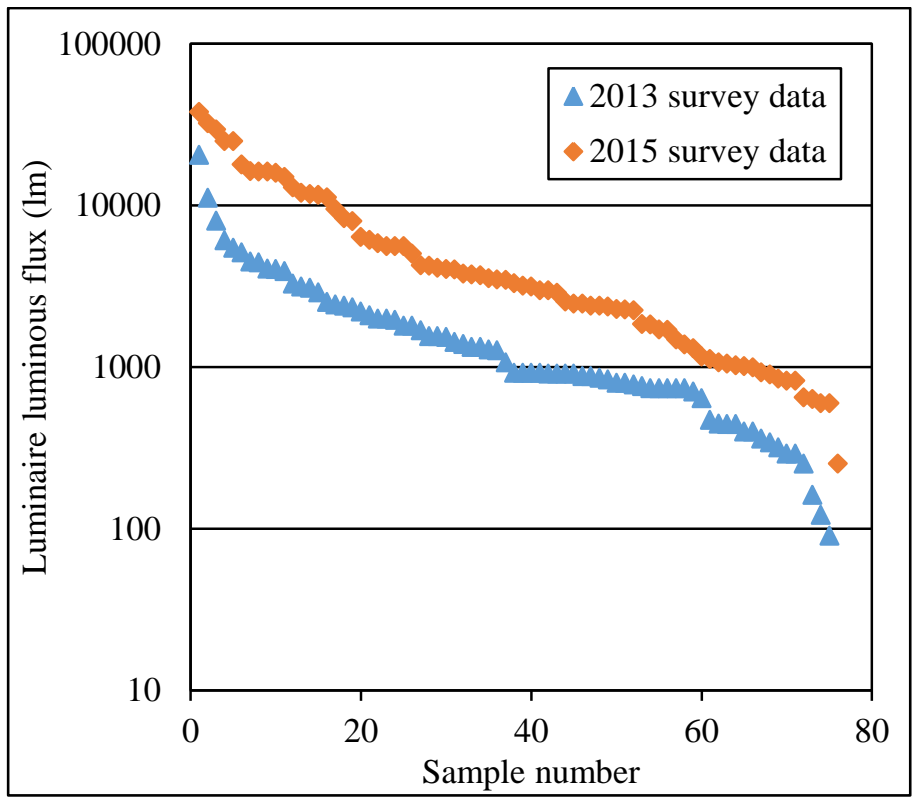

Fig. 6: Comparison of luminaire luminous flux survey data.

COB array based luminaires appeared to be a growing category. The aesthetic and physical constraints which are believed to promote the use of this type of light source are not expected to disappear, and cannot be offered by low and midpower components which require multiple LEDs to deliver equivalent output. Their ability to produce high luminous flux also makes them well suited to the ongoing drive for increased luminaire output, although there are practical limits to this factor. Therefore luminaires designed around COB modules are anticipated for the foreseeable future. Dedicated thermal management of these light sources remained relatively common. However, the survey data also indicated a growing proportion of these components avoid dedicated thermal management, possibly enabled by improving robustness or less demanding operating conditions. Therefore the demand for high performance thermal management techniques appears to be diminishing. Nonetheless, the majority of these systems employed a passive heatsink. Enhancing the performance of these systems still holds considerable commercial value. It may allow active cooling technologies and their drawbacks to be completely avoided, permit more compact luminaire designs, reduce costly and environmentally damaging material content, or improve lifecycle performance.

As the implementation of LEDs in luminaires continues to evolve this interpretation of results may prove to be invalid. The limitations of the study also produced questionable results. It is conceivable the survey samples were not truly representative of the wider industry thus distorting the apparent transition towards alternative LED categories and thermal management strategies. Therefore it is not possible to accurately quantify the changes occurring. However, every effort was made to conduct the work in a consistent and transparent manner so the findings are still believed to be valid. Based on the findings and expectations of the industry it is the authors' belief that major contradictions to 
this work are unlikely. Therefore these conclusions are offered with a reasonable degree of confidence.

\section{Conclusions}

The findings of this investigation suggest luminaires are moving away from high power LED packages towards more efficient or higher output alternatives. Mid-power LEDs appeared to be less reliant on dedicated heatsinks or other devices to maintain suitable operating conditions. Luminaires employing these components were therefore believed to be cheaper and easier to develop. This strategy met luminaire luminous flux demands while offering superior luminous efficacy, strengthening its appeal. Growing emphasis on lifecycle performance is expected to further enhance the adoption of mid-power LEDs and their associated thermal management techniques. On the other hand, the compact form of COB array type packages offered physical and aesthetic advantages which suit alternative applications. Their adoption is also supported by their high output which complements ongoing luminaire luminous flux increases. This maintains some future demand for high performance thermal management strategies However, there are indications that even for these typically high power components thermal management demands are relaxing as shown by the increasing proportion of luminaires which avoid dedicated management methods. Therefore future development around low cost, simple systems is believed to be more relevant to the lighting industry than high performance mechanisms for heat transfer.

\section{Acknowledgements}

Thanks go to Tamlite Lighting Ltd. for their unconditional support of, and contribution of resources towards, this investigation. Thanks are also owed to Thorlux Lighting and their technical manager P. Newman for providing product data upon the authors' request. This support had no bearing upon the outcomes of the research.

\section{References}

[1] R. Haitz and J. Y. Tsao, “Solid-state lighting: 'The case' 10 years after and future prospects.” Physica Status Solidi (A), vol. 208, no. 1, pp.17-29, Jan. 2011.

[2] McKinsey and Company Inc., "Lighting the way:

Perspectives on the global lighting market.” McKinsey and Company, 2nd ed. Aug. 2012.

[3] M. H. Chang et al., "Light emitting diodes reliability review.” Microelectronics Reliability, vol. 52, no. 5, pp.762782. May 2012.
[4] Tridonic, "Data Sheet TALEXmodule STARK FLE GEN1 12/15-LED152-4” [Online], Jan. 2016. Available from: http://www.tridonic.com. Accessed: Mar. 2016

[5] J. Pryde et al., “A Review of LED Technology Trends and Relevant Thermal Management Strategies.” 2014 IEEE Intersociety Conf. on Thermal and Thermomechanical Phenomena in Electronic Systems (ITherm), pp.31-38. May 2014.

[6] A. Christensen and S. Graham, "Thermal effects is packaging high power light emitting diode arrays.” Applied Thermal Engineering, vol. 29, no. 2-3, pp.364-371. Feb. 2009. [7] Samsung, "Data Sheet - LH351B” [Online], Rev. 7.10, Dec. 2015. Available from:

http://www.samsung.com/global/business/led/, Accessed: Dec. 2015.

[8] Samsung, "Data Steet - LM561B” [Online], Rev. 8.0, Feb. 2015. Available from:

http://www.samsung.com/global/business/led/, Accessed: Dec. 2015.

[9] Projecting Long Term Lumen Maintenance of LED Light Sources + Addendum B, Illuminating Engineering Society Standard TM-21-11, 2011.

[10] Samsung, "Samsung LM561B LED Package Completes LM-80 Testing for High Lumen Maintenance” [Online], Oct. 2013. Available from:

http://www.samsung.com/global/business/led/support/newsevents/news-detail?searchText=lm-

80\&startDate $=$ \&endDate $=$ \&page $=1$ \&rows $=24$ \& contentsId $=261$, Accessed: Mar. 2016.

[11] LG Innotek, "LG Innotek Announces High Power LED Packages With Best Efficacy” [Online], Sept. 2015. Available from:

http://www.lginnotek.com/community/news_view.jsp?seq=587,

Accessed: Mar. 2016.

[12] Thorn Lighting, "Environmental Product Declaration (EPD) certificates.” [Online], Available from:

http://www.thornlighting.co.uk/, Accessed: Dec. 2015.

[13] United States of America, Department of Energy, "LifeCycle Assessment of Energy and Environmental Impacts of LED Lighting Products. Part 1: Review of the Life-Cycle Energy consumption of Incandescent, Compact Fluorescent, and LED Lamps. Part 2: LED Manufacturing and Performance. Part 3: LED Environmental Testing.” Building Technologies Program, Aug. 2012. 